\title{
DOSSIER
}

\section{el LUGar de la teoría en la CONSTRUCCIÓN del OBJeto de INVESTIGACIÓN. DECISIONES TeÓRICAS QUE INVOLUCRAN UN CAMBIO EPISTEMOLÓGICO'}

\section{THE PLACE OF THEORY IN THE CONSTRUCTION OF THE RESEARCH OBJECT. THEOREIICAL DECISIONS INVOLVING AN EPISTEMOLOGICAL CHANGE}

Maria Florencia Di Matteo Demirdjian ${ }^{3}$

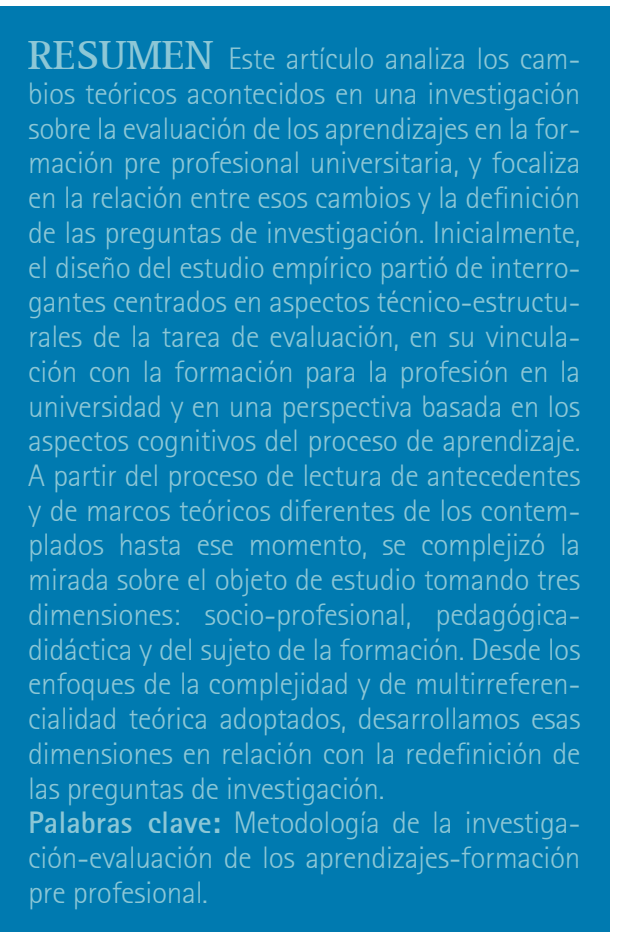

D01: http://dx.doi.org/10.30972/dpd.792808
1 Artículo Recibido: 4 de Septiembre 2017 - Aceptado: 5 de diciembre 2017

${ }^{3}$ Licenciada y Profesora en Ciencias de la EducaciónUBA. Magíster en Didáctica-UBA. Docente de Didáctica UBA- UNLu. Correo: florenciadm@hotmail.com

${ }^{4}$ Agradezco la lectura crítica y comentarios de la doctora Diana Mazza sobre el manuscrito original.

${ }^{5}$ Una versión de este trabajo fue presentada en el $12^{\circ}$ Congreso Nacional de Estudios del Trabajo El trabajo en su laberinto, viejos y nuevos desafíos. Asociación Argentina de Especialistas en Estudios del Trabajo (Aset).

${ }^{6}$ La investigación corresponde a una tesis de doctorado de la Facultad de Filosofía y Letras de la Universidad de Buenos Aires, dirigida por la doctora Diana Mazza.

\section{INTRODUCCIÓN ${ }^{4}$}

La investigación puede concebirse como una práctica social anclada en un contexto sociohistórico y originada en la problematización de algún aspecto de la realidad. Esta práctica supone trabajar con el entrecruzamiento de aspectos teóricos y empíricos a fin de construir conocimiento científico que permita ampliar la comprensión de esa realidad. En este proceso, el marco teórico-epistemológico adquiere un lugar central, ya que brinda perspectivas, teorias y conceptos que forman parte de la investigación, desde la elección del objeto del estudio y la definición de preguntas de investigación hasta el proceso de análisis y la elaboración de conclusiones. En otros términos, las teorias tienen y poseen implicancias metodológicas (Sautu, 2003). Dentro del proceso metodológico destacamos también la importancia de las preguntas de investigación en tanto permiten focalizar situaciones problemáticas y preocupaciones del investigador. Asimismo, las preguntas de investigación tienen un rol orientador al permitir visualizar el eje de la investigación.

Este artículo ${ }^{5}$ describe y analiza los cambios teóricos y epistemológicos experimentados en una investigación doctoral en curso y focaliza, especialmente, en la relación entre esas modificaciones y la definición de las preguntas de investigación. Se trata de un estudio sobre la evaluación de los aprendizajes en cursos universitarios de formación pre profesional en diferentes áreas del conocimiento, y analiza las vinculaciones de esta con características de la profesión y con el proceso formativo del sujeto ${ }^{6}$.

En la definición inicial del objeto problema de la investigación se emplea un marco teórico de base cognitivista y constructivista que aborda la evaluación desde sus aspectos técnico-instrumentales y al aprendizaje desde variables cognitivas, tomando especialmente líneas teóricas de origen anglosajón. Se trata de un marco teórico constituido por formaciones discursivas devenidas en saberes y luego ciencias (Foucault, 1970) que adquieren carácter hegemónico dentro del campo de la didáctica. Alli se inscribe inicialmente nuestro recorrido por la investigación que se relata en este trabajo. El proceso de redefinición del objeto problema recoge lecturas de 
De Prácticas y discursos

Universidad Nacional del Nordeste

Centro de Estudios Sociales

Año 7, Número 9, 2018, Marzo

ISSN 2250-6942

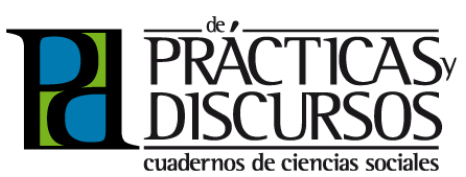

ABSTRACT This article analyzes the theo-

retical changes during a research about lear-

ning assessment in pre-professional training at

university level. It focuses on the relationship

between these changes and defining research

questions. Initially, the design of the empirical

study began with; questions focused on tech-

nical and structural aspects of the assessment

task, the connection with the professional tra-

ining, and a perspective based on the cognitive

aspects of the learning process. The studying

of the different background and theoretical fra-

meworks allows a complex view of the object of

study. It takes into account three dimensions:

socio-professional, pedagogic and didactic, and

the subject of learning. From the complexity and

multirreferencial theoretical approaches adop-

ted, we developed these dimensions in relation

to the redefinition of the research questions.

Key Words: Methodology of research-learning

assessment-pre-professional training.

\begin{abstract}
${ }^{7}$ El trabajo de investigación se denominó El uso de casos y problemas auténticos en la evaluación de los aprendizajes en la universidad. Su relación con las disposiciones hacia el aprendizaje y las modalidades de estudio de los alumnos. La tesis fue defendida el 16/12/2013 en la Facultad de Filosofía y Letras de la Universidad de Buenos Aires.
\end{abstract}

\footnotetext{
${ }^{8}$ Debido a la preocupación e interés de la tesis de Maestría, la teoría de Evaluación Auténtica (Wiggins, 1990) adquirió relevancia tanto en la definición de las preguntas de investigación como en las instancias de análisis de los datos. En el punto siguiente nos referimos a sus presupuestos teóricos.
}

diferentes investigaciones y marcos teóricos didácticos de origen francés y de otras disciplinas que posibilitan ampliar la mirada del objeto de estudio, incluyendo otras dimensiones en un sentido del construir nuevas figuras del pensar (Najmanovich, 2008). Se trata de un cambio teórico que implica también un viraje epistemológico para la investigación misma.

Este artículo se estructura de la siguiente manera. Inicialmente, se presenta el punto de partida de la investigación doctoral: los resultados de un estudio empírico previo -una tesis de maestría- y el análisis de los presupuestos epistemológicos de la Evaluación Auténtica (Wiggins, 1990), teoría que adquirió relevancia en el trabajo de maestría y en la definición inicial de las preguntas de investigación de la tesis doctoral. Luego se refiere a las preguntas de investigación tal como fueron redefinidas y las principales caracteristicas del enfoque metodológico adoptado en el estudio en curso. En tercer lugar, se presentan las conceptualizaciones teóricas que formaron parte del proceso de redefinición del objeto y las preguntas de investigación, explicitando cómo cada teoría ayudó en esa redefinición. Finalmente, se expone una sintesis y las conclusiones enfatizando la relación dialéctica existente entre el cambio teórico y epistemológico de la investigación y la construcción de las preguntas y el objeto de investigación en el marco de un enfoque de complejidad (Najmanovich, 2008) que habilita nuevas maneras de mirar.

\section{EN LOS INICIOS DE LA INVESTIGACION DOCTORAL: UN ESTUDIO PREVIO Y UN ANÁLISIS EPISTEMOLÓGICO}

El inicio de la investigación doctoral sobre la evaluación de los aprendizajes en la formación pre profesional universitaria se sitúa en las conclusiones elaboradas en el marco de una tesis de Maestría en Didáctica ${ }^{7}$. Desde ella estudiamos el uso de casos y problemas auténticos ${ }^{8}$ (Wiggins, 1990) en la universidad y su vinculación con modalidades de estudio de los alumnos.

Entre los objetivos de la tesis de Maestría situamos la descripción de los rasgos que asumen los casos y problemas auténticos como estrategias de evaluación, adoptados por cuatro profesores universitarios de las carreras de Derecho, Licenciatura en Administración y Licenciatura en Planificación y Diseño del Paisaje de las Facultades de Derecho, Ciencias Económicas y Arquitectura, Diseño y Urbanismo, y Agronomía de la Universidad de Buenos 
De Prácticas y discursos

Universidad Nacional del Nordeste

Centro de Estudios Sociales

Año 7, Número 9, 2018, Marzo

ISSN 2250-6942
El lugar de la Teoría en la construcción del objeto de Investigación.

Decisiones teôricas que involucran un cambio epistemológico

The place of Theory in the construction of the Research Object. Theoretical decisions involving an epistemological change

\footnotetext{
${ }^{9}$ El concepto "formato de evaluación" refiere a la forma que adopta la evaluación incluyendo la técnica e instrumento de evaluación, la demanda cognitiva, el grado de complejidad, la relación con la vida real y la práctica profesional. Se describe su modalidad de resolución: individual/grupal, en clase/fuera de ella, entrega inmediata/diferida (Camilloni y Cols, 2010).
}

Aires. Nos interesamos también por conocer la relación entre los formatos de evaluación ${ }^{9}$ basados en casos y problemas auténticos y las demandas cognitivas requeridas desde ellos, como así también la vinculación de esas modalidades de evaluación con las prácticas de enseñanza. La descripción de las percepciones de los estudiantes y sus modalidades y estrategias de estudio adoptadas para la preparación de los exámenes fue otro de los objetivos. El estudio empírico se llevó a cabo mediante un estudio de casos desde una estrategia metodológica cualitativa.

Las conclusiones de la tesis de Maestría indicaron una coherencia o alineación entre las prácticas de enseñanza y las de evaluación, y una articulación entre ellas y una concepción de la disciplina que se enseña y de la profesión para la que forman. Los casos y problemas auténticos presentaron diferentes grados de extensión, provinieron de diferentes fuentes y tuvieron variados grados de autenticidad según su cercanía o lejanía respecto de la práctica real. Los instrumentos de evaluación contenían consignas de producción o elaboración de respuestas y, en menor medida, preguntas de verdadero o falso a justificar y otras de desarrollo conceptual. Desde estos formatos de evaluación se requirió poner en juego procesos cognitivos intermedios y superiores: el uso y aplicación de estrategias cognitivas generales (justificar utilizando la teoría, sacar conclusiones, jerarquizar información) y específicas de cada dominio disciplinar.

Desde la perspectiva de los estudiantes, la modalidad de evaluación consistió en analizar casos prácticos o resolver situaciones problemáticas empleando conocimientos disponibles, caracterización coincidente con la descripción del formato de evaluación. Ellos destacaron que este tipo de evaluación permitió situarlos en la profesión, promovió un aprendizaje comprensivo, favoreció demostrar lo aprendido, aplicar y reflexionar sobre los conceptos, y propiciar una articulación entre la teoría y la práctica. La modalidad de estudio adoptada, según señalaron los mismos estudiantes, fue continua a través de las clases, individual, comprensiva, centrada en lo práctico y en la aplicación de nociones teóricas.

En cuanto a las actividades de preparación para la evaluación, los estudiantes refirieron a la lectura del material bibliográfico, la resolución de guias o actividades dadas en clase, el tomar en cuenta lo señalado como importante por el profesor y la focalización en la relación entre textos y clases. Según estos datos, observamos como tendencia una orientación hacia un enfoque 
De Prácticas y discursos

Universidad Nacional del Nordeste

Centro de Estudios Sociales

Año 7, Número 9, 2018, Marzo

ISSN 2250-6942

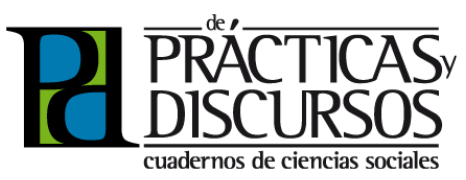

de aprendizaje profundo ${ }^{10}$ (Marton y Saljö, 1976).

En cuanto a la relación entre formato de evaluación y modalidad de estudio de los alumnos participantes de esta investigación, encontramos que esta última se acerca, en cuanto a demanda cognitiva, a lo requerido desde los formatos de evaluación. Teniendo en cuenta la coherencia observada entre las prácticas de enseñanza y las de evaluación, si los alumnos estudian considerando los requerimientos de la clase y del docente, tal como lo indican ellos mismos, entendemos que también estarian estudiando, tomando en cuenta las demandas de la evaluación.

La elaboración de estas conclusiones de la tesis de Maestría abrió algunos interrogantes que formaron parte de las preguntas de origen de la investigación doctoral. En la definición inicial, el interés residió en continuar estudiando la evaluación de los aprendizajes a través de casos auténticos (Wiggins, 1990), pero como expresión de formas de educación experiencial $(K o l b, 2001)^{11}$, añadiendo la dimensión emocional del sujeto desde sus actitudes y disposiciones hacia el estudio y la profesión. Las siguientes fueron las preguntas del proyecto de doctorado en su estado inicial:

- ¿Qué instrumentos de evaluación se utilizan en las experiencias de formación profesional a través de la educación experiencial?

- ¿Cuáles son los rasgos que asumen modalidades de evaluación de los aprendizajes en propuestas de educación experiencial a través de casos auténticos de la práctica profesional?

- ¿Cuáles son las características de los casos como formas de evaluación de los aprendizajes en propuestas de evaluación experiencial en lo referente a sus propósitos, su formato y las condiciones de puesta en práctica?

- ¿Cuál es la concepción de formación para la profesión que subyace en la elección de las prácticas e instrumentos de evaluación?

- ¿En qué grado se consideran los saberes del perfil del graduado presentes en los planes de estudio para la construcción de las prácticas e instrumentos de evaluación?

- ¿Qué habilidades, disposiciones y actitudes de los alumnos en relación con el estudio y con la profesión son requeridas desde estos formatos de evaluación?

- ¿Cuáles son las percepciones y opiniones de los estudiantes sobre estas prácticas e instrumentos de evaluación y su vinculación con las actitudes hacia el estudio y la profesión? 
De Prácticas y discursos

Universidad Nacional del Nordeste

Centro de Estudios Sociales

Año 7, Número 9, 2018, Marzo

ISSN 2250-6942
El lugar de la Teoría en la construcción del objeto de Investigación.

Decisiones teốricas que involucran un cambio epistemológico

The place of Theory in the construction of the Research Object. Theoretical decisions involving an epistemological change

Estos interrogantes pretendian ampliar la mirada de la evaluación -desde el instrumento y su relación con la enseñanza- al situarla en el marco de la formación para la profesión, focalizando en casos de evaluación con un alto grado de autenticidad y concibiendo al sujeto de la formación desde lo emocional, además desde lo cognitivo y racional. En estas preguntas, el concepto de autenticidad ocupaba un lugar central. Debido a ello, nos propusimos analizar los presupuestos epistemológicos de la teoría de Evaluación Auténtica (Wiggins, 1990), tarea que despertó una serie de interrogantes.

Uno de los presupuestos de la mencionada teoría radica en el interés por examinar el desempeño de los estudiantes a través de tareas auténticas, es decir, mal definidas, con límites borrosos y múltiples posibilidades de respuesta. En cuanto al tipo de trabajo demandado, se espera que el estudiante analice y resuelva tareas poco estructuradas que lo ayuden a abordar complejidades y ambigüedades de la vida real y profesional. Aquí nos preguntamos ¿cuál es el criterio desde el que esta teoría define a lo real?, ¿la realidad en sí?, ¿una representación de ella?, ¿de qué tipo? Tomando la noción de criterios y referentes que orientan la construcción del juicio de valor constitutivo de toda evaluación (Ardoino y Berger, 1998), nos interrogamos ¿cuáles son los criterios de evaluación que permiten analizar los datos de la evaluación en las Ilamadas prácticas de evaluación auténtica?, ¿es la realidad externa en sí?, ¿una representación de la realidad? Esos referentes, ¿surgen de las prácticas profesionales, de las prácticas de enseñanza, de las de formación o de las del proceso del sujeto que aprende? Encontramos así un conjunto de preguntas de orden epistemológico para los que la teoría de Evaluación Auténtica no presenta indicios de respuesta.

Otro de los aspectos que problematizamos fue la naturaleza de los aprendizajes que se propone promover según la teoría de Evaluación Auténtica (Wiggins, 1990) y, junto con ello, el modo de concebir a quienes aprenden. De acuerdo con el análisis de esta teoria, encontramos que el énfasis se coloca en la puesta en juego de conocimientos y procedimientos para el análisis de casos y situaciones problemáticas, y la elaboración de proyectos, en la movilización de actitudes de búsqueda de información y en la predisposición de resolver problemas. Estas tareas que forman parte de la propuesta de enseñanza y de evaluación enfatizan un trabajo sobre los contenidos en profundidad antes que en exten- 
De Prácticas y discursos

Universidad Nacional del Nordeste

Centro de Estudios Sociales

Año 7, Número 9, 2018, Marzo

ISSN 2250-6942

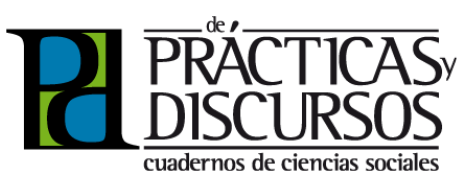

sión. Implican una tarea de integración conceptual, pero también desde las estrategias y las disposiciones y actitudes (Ahumada, 2005). Inferimos de alli una perspectiva del aprendizaje que incluye aspectos de la vida emocional del sujeto al referir a la motivación, la adquisición de procedimientos, la comprensión y los logros. Sin embargo, se trata de una mirada que enfatiza los aspectos racionales y cognitivos del sujeto de aprendizaje.

De acuerdo con el análisis realizado, sostenemos que la teoría de Evaluación Auténtica (Wiggins, 1990) no presenta un posicionamiento ni una reflexión epistemológica clara ni explícita. Teniendo en cuenta la importancia que asume el marco epistemológico en toda investigación, emprendimos la búsqueda de otras teorías que permitieran dialogar con aquella y ampliar la mirada sobre diferentes dimensiones del objeto de estudio. De esta manera, comenzamos con una revisión de antecedentes referidos a la formación profesional en la universidad. Entre ellas encontramos dos trabajos que tomaron en su abordaje aspectos socioprofesionales, institucionales, pedagógicos, grupales y del sujeto de formación (Andreozzi, 2006; Souto y otros, 2010), incluyendo aportes de líneas teóricas francesas. Estas dimensiones contribuyeron a tomar conciencia de la necesidad de complejizar la perspectiva sobre la evaluación en la formación profesional universitaria, mirándola con nuevos ojos (Najmanovich, 2008), y pensar en redefinir las preguntas de investigación. En este marco, con la explicitación de otras inquietudes, iniciamos esa redefinición de las preguntas iniciales de la investigación doctoral en un trabajo de ida y vuelta con la lectura de otras teorias.

\section{LA INVESTIGACIÓN DOCTORAL Y SUS REDEFINICIONES: LA RELACIÓN ENTRE LAS PREGUNTAS DE INVESTIGACIÓN Y LOS EFECTOS DE LA REDEFINICIÓN TEÓRICA EN LAS PREGUNTAS DE INVESTIGACIÓN}

De acuerdo con los análisis realizados, según señalamos en el apartado anterior, decidimos redefinir las preguntas iniciales de la investigación doctoral. Ese proceso de cambios teóricos quedó expresado a través de los siguientes interrogantes:

- ¿Qué rasgos asumen los dispositivos de formación y de evaluación de los aprendizajes, empleados en una selección de cursos universitarios de formación pre profesional en lo relativo a fundamentos pedagógicos y didácticos, sus propósitos, 
De Prácticas y discursos

Universidad Nacional del Nordeste

Centro de Estudios Sociales

Año 7, Número 9, 2018, Marzo

ISSN 2250-6942
El lugar de la Teoría en la construcción del objeto de Investigación. Decisiones teốricas que involucran un cambio epistemológico

The place of Theory in the construction of the Research Object. Theoretical decisions involving an epistemological change

\footnotetext{
${ }^{12}$ Es posible realizar una diferenciación al interior del enfoque clínico: entendido en sentido amplio o restringido (Souto, 2010b). Mientras este último implica el empleo del psicoanálisis de manera exclusiva, el sentido amplio toma al psicoanálisis junto con otros enfoque teóricos.
}

formatos y condiciones de puesta en práctica?

- ¿Cuáles son las concepciones sobre la práctica profesional y su posible vinculación con las situaciones de formación para la profesión que subyacen en cada curso?

- ¿Qué lugar ocupan la profesión y las caracteristicas del mundo del trabajo en la definición conceptual y operativa de las prácticas e instrumentos de evaluación de los aprendizajes utilizados, en el marco de las instancias de formación a estudiar? - ¿Cómo se caracteriza la relación que el sujeto establece con la situación de formación en general y con la instancia de evaluación en particular a partir de aspectos tales como: las habilidades cognitivas implicadas en el desempeño en la situación, la vinculación emocional establecida, las representaciones sobre la formación, la evaluación y la profesión como práctica futura del estudiante de evaluación?

En términos metodológicos, el estudio se enmarca en el enfoque clínico en sentido amplio (Souto, 2010b; Mazza, 2015), desde una lógica cualitativa y una perspectiva hermenéutica. La metodología asume un estudio de casos que, por su carácter particularistico y descriptivo (Sautu, 2003), permite estudiar en profundidad las singularidades de la situación en estudio. Los casos seleccionados corresponden a cursos de carreras de diferentes áreas del saber: medicina, derecho, ingeniería y trabajo social. Todos pertenecen a universidades públicas de la Argentina.

Desde un punto de vista epistemológico, para este enfoque, la construcción del conocimiento implica la elaboración de hipótesis interpretativas con una finalidad comprensiva antes que explicativa. Esa interpretación se entiende como una construcción de sentidos por parte del investigador sobre las situaciones de estudio que implican a sujetos individuales o colectivos. Debido a ello, este enfoque otorga una centralidad a la relación intersubjetiva que se genera entre sujeto y objeto de la investigación, especialmente reconociendo la presencia del inconsciente y de las relaciones de transferencia y contratransferencia. Para referirnos a ello, utilizamos el concepto de implicación (Barbier, 1977; Ardoino, 1997). Dentro de este enfoque clínico en sentido amplio'2 (Souto, 2010b), tomamos diferentes marcos teóricos para la redefinición de las preguntas de investigación y para la posterior interpretación y el análisis de los datos. La adopción de este enfoque de multirreferencialidad teórica (Ardoino, 1993) permitió construir el objeto evaluación de los aprendizajes en situación de formación pre profesional con- 
De Prácticas y discursos

Universidad Nacional del Nordeste

Centro de Estudios Sociales

Año 7, Número 9, 2018, Marzo

ISSN 2250-6942

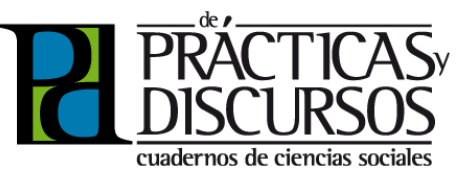

siderando distintos planos de significación y articulando diferentes miradas sobre el fenómeno, pero sin pretensión de síntesis.

Desde este enfoque y tal como hemos mencionado más arriba, la lectura de las teorias enmarcadas en el proceso de redefinición del marco conceptual permitió advertir distintas dimensiones del objeto de estudio, entre las que situamos las socioprofesionales, la pedagógico- didáctica, la instrumental y la del sujeto de la formación. Esta perspectiva representa una variación respecto de investigaciones previas en el tema (Lipsman, 2004; Camilloni y otras, 2012; Flores, 2012; Di Matteo, 2013, 2014; Finkelstein, 2016), las que asumen un abordaje constructivista y cognitivista de la evaluación de los aprendizajes.

Desde una dimensión socioprofesional, nos interesamos por comprender las relaciones entre la formación pre profesional universitaria y las características del mundo del trabajo. La pedagógica-didáctica permite focalizar en los fundamentos pedagógicos y didácticos de la propuesta de evaluación, la concepción de la práctica y del saber profesional. La dimensión instrumental posibilita analizar la evaluación desde nociones tales como tiempos, espacios, tareas, actividades, formato de evaluación, entre otros. Finalmente, desde la dimensión del sujeto de formación, nos proponemos comprender las habilidades cognitivas implicadas en las situaciones de formación y evaluación, su vinculación emocional con el conocimiento, sus representaciones sobre la situación de formación, la evaluación y la futura práctica profesional.

\section{LAS TEORÍAS ADOPTADAS EN LA REDEFINICIÓN DEL OBJETO Y PREGUNTAS DE INVESTIGACIÓN DOCTORAL}

En este apartado nos referimos a algunas conceptualizaciones teóricas adoptadas para complejizar la mirada sobre las prácticas de evaluación en instancias de formación pre profesional en la universidad. Aquí, nos referimos a la formación en términos generales y a la formación pre profesional en lo particular, a la profesión y al campo laboral, a concepciones sobre la evaluación de los aprendizajes y al sujeto de la formación.

\section{ACERCA DE LA FORMACIÓN}

Una primera problematización sobre las prácticas pre profesionales nos llevó a considerar una perspectiva de la formación en- 
De Prácticas y discursos

Universidad Nacional del Nordeste

Centro de Estudios Sociales

Año 7, Número 9, 2018, Marzo

ISSN 2250-6942
El lugar de la Teoría en la construcción del objeto de Investigación.

Decisiones teốricas que involucran un cambio epistemológico

The place of Theory in the construction of the Research Object. Theoretical decisions involving an epistemological change

tendida como un trabajo sobre sí mismo que requiere de mediaciones, soportes y condiciones para su realización (Ferry, 2008). Analizamos la perspectiva de la enseñanza (Hirst, 1977; Fenstermacher, 1989), entendida como actividad intencional cuya finalidad es producir aprendizajes en un otro, que inicialmente carece de él. Se trata de un posicionamiento que sitúa al docente en un lugar central de transmisor de contenidos. "El término enseñar o enseñanza es la etiqueta que ponemos a aquellas actividades de una persona $A$, que tiene la intención de producir en otra persona, $B$, el aprendizaje intencional de $X$ (creencias, actitudes, destrezas)" (Hirst, 1977: 312).

A diferencia de la perspectiva de la enseñanza, desde nuestro posicionamiento entendemos que la formación no es algo que se recibe, sino que es el sujeto quien se forma, encuentra su forma y dinámica de desarrollo personal. De este enfoque no acordamos con la noción según la cual "alguien forma a alguien"; es el mismo sujeto "quien se forma" o, dicho de otro modo, "encuentra su forma, es él quien se desarrolla, diría, de forma en forma" (Ferry, 2008:54).

En esta perspectiva, la formación requiere de variadas mediaciones que pueden ser: las lecturas, los encuentros con otros, las diferentes circunstancias. Se trata de un proceso que presupone ciertos soportes o medios tales como lo son la tarea de enseñar y el proceso de aprender, el plan de estudios, los objetivos de aprendizaje, los recursos, entre otros. La formación requiere también de determinadas condiciones: un tiempo y un lugar para reflexionar y realizar ese trabajo sobre uno mismo. Entre estas se ubica también la toma de distancia con la realidad, situación que posibilita desprenderse de ella para representarla y de esta manera poder abordarla (Ferry, 2008).

La formación entendida como un trabajo sobre si mismo implica, entonces, reflexionar y revisar aquello realizado y pensando en otras maneras de pensar y actuar (Ferry, 2008). Asi, formarse es adquirir una forma de actuar, de pensar y de perfeccionar esa forma. Este recorrido no acontece con el mero hacer, sino con el volver(se), rever(se) y reflexionar sobre aquello realizado. "Reflexionar es al mismo tiempo reflejar y tratar de comprender, y en ese momento sí hay formación" (Ferry, 2008:56). Esta perspectiva de la formación permite pensar el lugar de las diferentes prácticas e instancias de evaluación como parte de un proceso que posibilita esa toma de distancia de las prácticas pre profesionales en un espacio laboral real. En este sentido, esta con- 
De Prácticas y discursos

Universidad Nacional del Nordeste

Centro de Estudios Sociales

Año 7, Número 9, 2018, Marzo

ISSN 2250-6942

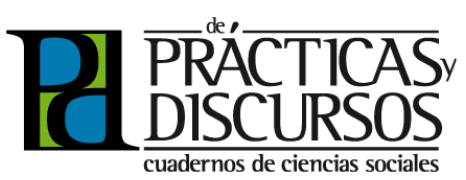

\begin{abstract}
${ }^{13}$ Existen diferentes perspectivas teóricas sobre la noción de competencias. Si bien no es objeto de este trabajo desarrollar los diferentes enfoques, señalamos brevemente que este concepto ingresa al sistema educativo desde el mundo laboral. Desde mediados de los 90 comienzan a surgir, para los distintos niveles del sistema educativo, múltiples referencias a las competencias: "formación por competencias", "planes de estudio basado en el enfoque de competencias", "propuestas educativas por competencias". Esta adopción del término no se da sin dificultades, pues genera, según Barnett (2001), angustias en dos sentidos: por un lado, por la migración de valores de otros medios hacia el ámbito académico $y$, por otro, a partir de la disminución de la libertad académica y de autonomía institucional como rasgo histórico distintivo de las universidades. El concepto ha sido puesto en debate nuevamente, a partir de los procesos de cambio curricular acaecidos en el Nivel Superior en la Unión Europea.
\end{abstract}

ceptualización de la formación amplía la noción de evaluación más allá del instrumento y la liga con concepciones epistemológicas y éticas sobre el sujeto de la formación. En términos de las preguntas de investigación, tal como quedaron redefinidas, se vincula con aquellas que abordan a la evaluación en sus relaciones con el dispositivo de formación desde los fundamentos pedagógicos y didácticos, y las que se interesan por estudiar la relación que establece el sujeto con la situación de formación en términos generales.

\section{SOBRE LA FORMACIÓN PRE PROFESIONAL}

Para caracterizar a la formación pre profesional, partimos de la diferenciación entre los mundos de la enseñanza, la formación y la profesión, entendidos como modelos teóricos (Barbier, 1999; Barbier y Galatanu, 2004). El mundo de la enseñanza es aquel que se centra en la transmisión del contenido a fin de lograr que los estudiantes se apropien de él. Se trata de una perspectiva ligada con la enseñanza entendida como actividad intencional (Hirst, 1977; Fenstermacher, 1989). El mundo de la formación, en cambio, focaliza en el desarrollo de capacidades, habilidades y actitudes del sujeto de la formación. Esta idea permite pensar en situaciones que requieren de operar con ciertas destrezas, capacidades sobre algo del orden de la realidad. Estas referencias teóricas posibilitan pensar que los conocimientos involucrados en la evaluación de los aprendizajes en la formación pre profesional se sitúan en el hacer tanto a nivel del pensamiento como de la acción. Desde el mundo de la profesión, el eje se coloca en el desarrollo de competencias ${ }^{13}$ que surgen en el mundo laboral. Desde aquí, entonces, la referencia es el mundo del trabajo. Esta noción complejiza aún más la construcción de nuestro objeto de estudio, ya que la finalidad última de la formación pre profesional es la preparación para el mundo laboral, el que requiere la puesta en acción de saberes en contextos de desempeño real. A fin de complejizar este análisis, consideramos las articulaciones y tensiones dadas entre esa práctica laboral real y la noción de artificiosidad (Souto, 2010a). En tanto, los primeros dos mundos describen y analizan situaciones de enseñanza y formación creadas artificialmente para favorecer el logro de los objetivos previstos en los estudiantes; el mundo de la profesión designa el desarrollo de saberes que surgen en el mismo ejercicio laboral. 
De Prácticas y discursos

Universidad Nacional del Nordeste

Centro de Estudios Sociales

Año 7, Número 9, 2018, Marzo

ISSN 2250-6942
El lugar de la Teoría en la construcción del objeto de Investigación.

Decisiones teốricas que involucran un cambio epistemológico

The place of Theory in the construction of the Research Object. Theoretical decisions involving an epistemological change

Estas conceptualizaciones nos permiten situar, en primer lugar, a los cursos de formación pre profesional en la convivencia entre los tres mundos. En términos de supuestos que operan en la definición del objeto concebimos, además, que los cursos de formación pre profesional presentarian, predominantemente, la intención de la formación y en menor medida, la de la enseñanza. En segundo lugar, estas nociones teóricas posibilitan tomar conciencia que esos cursos del estudio empírico tendrían rasgos de lo artificial (Souto, 2010a), es decir, sujetos siempre a tiempos y espacios, y a tareas y actividades creadas con una finalidad de formación que es artificial. En tercer lugar, estos conceptos ayudan a redefinir las preguntas de investigación en tanto interés por describir los rasgos de esos dispositivos de formación y de evaluación de los aprendizajes -sabiéndolos artificiales-, en cuanto a los fundamentos pedagógicos y didácticos, sus propósitos, formatos y condiciones de puesta en práctica. Asimismo, debido a las estrechas vinculaciones entre los tres mundos, nos interesamos por identificar el lugar que ocupa la profesión y las características del mundo del trabajo en la definición conceptual y operativa de las prácticas e instrumentos de evaluación de los aprendizajes utilizados, en el marco de las instancias de formación a estudiar.

Situada la formación pre profesional en el entramado de los mundos de la enseñanza, la formación y la profesión, avanzaremos en precisar modos de concebir la formación pre profesional. En términos generales, ubicamos a nuestro objeto de estudio en el nivel de educación superior universitaria, en el que la formación hacia la profesión es una de sus principales funciones.

Desde un punto de vista analítico, podemos diferenciar entre momentos de formación inicial o pre profesional y momentos de formación continua. El primero refiere a la formación brindada desde instituciones de formación de Nivel Superior (universidades, institutos de formación profesional, docente, técnico, etcétera). La formación continua es la que sucede en el marco del ejercicio profesional. La formación intenta lograr la profesionalidad en tanto articulación singular de saberes y actitudes que permiten al individuo actuar en un campo (Souto y otros, 2010), o sea: "en un espacio social que goza de una autonomía relativa y que es socialmente reconocido como especializado en la producción de cierto tipo de transformación de lo real" (Barbier, 1999:82).

Realizadas estas aclaraciones, definimos a las prácticas pre profesionales como aquellas que tienen por objeto desarrollar acti- 
De Prácticas y discursos

Universidad Nacional del Nordeste

Centro de Estudios Sociales

Año 7, Número 9, 2018, Marzo

ISSN 2250-6942

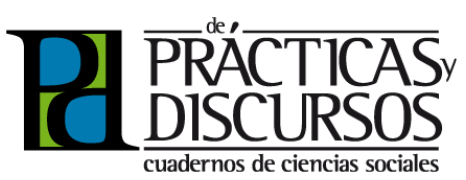

tudes, conocimientos y competencias que se requieren para ejercer un empleo o un oficio (lesalc-Unesco, 2006), y designan tipos de formación organizada que permiten desarrollar esas competencias o calificaciones necesarias para el ejercicio de esa actividad (Afpa, 1992). Según señala el Glosario Regional de América Latina, la formación profesional es el:

proceso teórico práctico sistemáticamente adelantado, mediante el cual las personas adquieren, mantienen o mejoran conocimientos técnicos y tecnológicos, destrezas, aptitudes y valores que sustentan su empleabilidad como trabajadores y su capacidad para actuar crítica y creativamente en la actividad productiva, contribuyendo así a su propio desarrollo personal, a la competitividad de la economía y al ejercicio pleno de la ciudadanía (Iesalc-Unesco, 2006: 317-318).

Asimismo, tomamos aportes de Souto y otros (2010), quienes definen a la formación pre profesional como el espacio formativo en el que se relacionan los mundos de la formación y del trabajo de un modo cercano, y en donde se desarrolla la profesionalización inicial de los sujetos. Se trata de un espacio que contribuye a la construcción de la identidad profesional que seguirá su construcción en el campo laboral.

En lo que respecta a la definición de nuestro objeto de estudio, las nociones desarrollas permitieron advertir que ciertos aspectos de la profesión y del mundo laboral estarian presentes, con distintos enfoques y grados, en las prácticas, estrategias y dispositivos de formación que ponen al estudiante en vinculación con el objeto de estudio, trabajo e intervención.

\section{LA PROFESIÓN Y EL CAMPO LABORAL}

Tal como hemos señalado, las prácticas pre profesionales se encuentran en un entramado entre los mundos de la enseñanza, la formación y la profesión (Barbier, 1999; Barbier y Galatanu, 2004) y presentan un alto grado de artificiosidad (Souto, 2010a). En este marco, la profesión, a la que se orientan estas prácticas de formación, ocupa un lugar relevante en el diseño de los dispositivos de formación, así como en las expectativas y representaciones de los estudiantes. De alli la importancia de definirla. Señalamos entonces que entendemos a la profesión en términos generales como un: "fenómeno socio-cultural que se constru- 
De Prácticas y discursos

Universidad Nacional del Nordeste

Centro de Estudios Sociales

Año 7, Número 9, 2018, Marzo

ISSN 2250-6942
El lugar de la Teoría en la construcción del objeto de Investigación.

Decisiones teốricas que involucran un cambio epistemológico

The place of Theory in the construction of the Research Object. Theoretical decisions involving an epistemological change

ye y se sitúa en un contexto socio-histórico y está sometida a transformaciones provenientes de la vida social, política, cultural y económica, del desarrollo del conocimiento y las tecnologías" (Souto y otros, 2010:4).

El estudio de la profesión implica tener en cuenta cómo afecta a su desarrollo el crecimiento y transformación del conocimiento en el marco de la expansión de las relaciones económicas, sociales y políticas en un sistema de producción capitalista. Esto constituye un fuerte estímulo para la especialización -a diferencia del conocimiento generalista- y aumenta la probabilidad de obsolescencia del profesional instalando la necesidad de actualización permanente; también puede dificultar el trabajo cooperativo entre distintos especialistas que tienen intereses, actitudes y lenguajes diferentes (Gómez Campo y Tenti Fanfani, 1993).

Tomando el concepto de campo de Bourdieu (1990), entendemos a las profesiones como espacios estructurados de posiciones ocupadas por miembros que pugnan por posiciones de poder. Específicamente, las concebimos como un caso particular de desarrollo general de campos estructurados de producción de bienes simbólicos en una sociedad (Bourdieu, 1973). Los profesionales producen servicios: bienes simbólicos al interior de espacios objetivos relativamente autónomos respecto del conjunto de la sociedad (Gómez Campo, y Tenti Fanfani, 1993).

Según lo señalado, las profesiones constituyen campos cuya definición es independiente de las caracteristicas y funciones de quienes los ocupan. Sus rasgos centrales son: las jerarquías (posiciones dominantes y dominadas), los límites (quienes pertenecen al campo y quienes no) y la autonomía (o la capacidad de interpretar las producciones externas conforme a sus propios principios). Esto permite pensar en la complejidad creciente del rol del profesional en la medida en que se inscribe dentro de espacios sociales que demandan interactuar con colegas de otras áreas disciplinares, otros especialistas, administradores y con clientes colectivos cuyos problemas no aparecen delimitados en forma clara e inmediata (Gómez Campo y Tenti Fanfani, 1993). Este modo de comprender a la profesión como campo (Bourdieu, 1990) y, como tal, constituida por luchas de poder, posibilita pensar, por ejemplo, en prácticas profesionales que son dominantes, unas emergentes y otras alternativas. En este sentido, coincidimos con Follari (2010) en señalar que no existe una, sino prácticas profesionales diferenciadas, y la formación para algu- 
De Prácticas y discursos

Universidad Nacional del Nordeste

Centro de Estudios Sociales

Año 7, Número 9, 2018, Marzo

ISSN 2250-6942

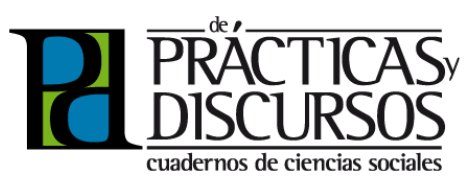

nas prácticas en detrimento de otras es una opción valorativa, es decir, no es neutral.

Estas nociones teóricas permitieron orientar la definición de los siguientes objetivos de investigación: caracterizar las concepciones sobre la práctica profesional y su posible vinculación con las situaciones de formación para la profesión que subyacen en cada curso, e identificar el lugar que ocupa la profesión y las características del mundo del trabajo en la definición conceptual y operativa de las prácticas e instrumentos de evaluación de los aprendizajes utilizados, en el marco de las instancias de formación a estudiar. Si la formación implica un trabajo sobre las representaciones de la realidad -y no la realidad en sí-, y una construcción de un personaje moldeado -con ciertas formas- según ciertas exigencias sociales, será preciso indagar sobre las nociones de los estudiantes -y también de los docentes-sobre la profesión y la práctica profesional futura. Esta indagación cobra mayor valor al considerar que esas representaciones actúan como mediadores en la transición entre la enseñanza, la formación y la profesión.

\section{SOBRE LA EVALUACIÓN DE LOS APRENDIZAJES}

El estudio de la evaluación de los aprendizajes implica situarla en la complejidad conceptual y práctica en la que se encuentra inmersa; es decir, considerarla como "objeto de estudio y campo de práctica" (Cols, 2009).

La evaluación es una práctica situada en un contexto social, en un marco institucional y en el pensamiento del docente. El análisis de las prácticas de evaluación requiere también del estudio de las concepciones epistemológicas sobre la formación y el conocimiento tanto del profesor como de los estudiantes. La evaluación de los aprendizajes como proceso educativo se vincula con las preguntas sobre qué enseñar y evaluar, cómo hacerlo y con qué finalidades. Las distintas formas de concebirla remiten a maneras particulares de entender al sujeto, al conocimiento, a las relaciones que entre ellos se establecen en una sociedad.

Para referirse a otras problemáticas que involucran al tema de la evaluación, Cols (2009) expresa que la evaluación se presenta como un tema controversial y evitado. Ella comenta que se encuentra tensionada por lógicas antagónicas: "evaluación de procesos-evaluación de productos", "evaluación con fines de certificación de aprendizajes-evaluación para mejorar los apren- 
De Prácticas y discursos

Universidad Nacional del Nordeste

Centro de Estudios Sociales

Año 7, Número 9, 2018, Marzo

ISSN 2250-6942
El lugar de la Teoría en la construcción del objeto de Investigación.

Decisiones teốricas que involucran un cambio epistemológico

The place of Theory in the construction of the Research object. Theoretical decisions involving an epistemological change

dizajes", "evaluación objetiva-evaluación subjetiva", entre otras. Para Perrenoud (2008), la evaluación se encuentra situada entre una lógica tradicional y otra emergente: "fabricar jerarquías de excelencia" (selección y jerarquización de estudiantes) o "regular los aprendizajes". Pero, más allá de estas tensiones, señala este mismo autor que la cuestión es aún más compleja.

Evidentemente resulta simplificador describir la evaluación como oscilante sólo entre dos lógicas. En realidad, hay en ella muchas otras, incluso más pragmáticas. Antes de regular los aprendizajes, la evaluación regula el trabajo, las actividades, las relaciones de autoridad y la cooperación en el aula y, por otro lado, las relaciones entre la familia y la escuela, o entre los profesionales de la educación. Una mirada sociológica intenta constantemente considerar a la vez las lógicas del sistema-que se refieren al tratamiento de las diferencias y las desigualdades-y las lógicas de los actores, que remiten a las apuestas más cotidianas de la coexistencia, de control, de poder. (Perrenoud, 2008:10).

Situar a la evaluación de los aprendizajes en el nivel de educación superior añade a su estudio otras complejidades. Por un lado, su carácter crítico, debido a la responsabilidad por la acreditación de competencias profesionales. Por otra, su atravesamiento por la aceleración del avance científico-tecnológico y el crecimiento $y$, finalmente, la diversificación de la población estudiantil.

Desde una mirada técnico-instrumental, señalamos que la evaluación constituye un juicio que se efectúa sobre un conjunto de informaciones que el profesor recoge acerca del proceso de aprendizaje de los estudiantes en vista a la toma de decisiones (Camilloni, 2010). Estas informaciones constituyen los datos de referencia de toda evaluación y condicionan la calidad de los juicios evaluativos. Esto conduce al problema de la definición de los instrumentos de evaluación.

Este abordaje conceptual de la evaluación implica, también, enfatizar que la recolección de datos no es una mera descripción, ya que esa información es puesta en relación con algún/os marco/s de referencia (Ardoino y Berger, 1998).

Las nociones sobre evaluación de los aprendizajes desarrolladas permiten pensar a la evaluación en el marco propositivo mayor de formación en la universidad, destacar el lugar que tiene en tanto certifica competencias para el ejercicio profesional, y tomar conciencia de los lugares que la profesión y la práctica 
De Prácticas y discursos

Universidad Nacional del Nordeste

Centro de Estudios Sociales

Año 7, Número 9, 2018, Marzo

ISSN 2250-6942

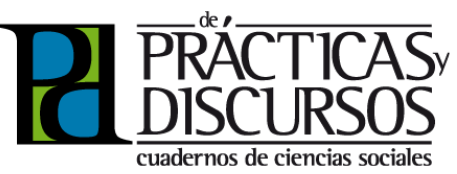

profesional pueden ocupar en la definición de prácticas, instrumentos y criterios de evaluación.

\section{SOBRE EL SUJETO DE LA FORMACIÓN}

La concepción de la formación como trabajo sobre uno mismo (Ferry, 2008) supone una concepción singular del sujeto. Formarse implica volver sobre las motivaciones, los miedos, los angustias, los temores y también sobre los aprendizajes técnicos logrados; se trata de una construcción subjetiva mediada siempre por el encuentro y el diálogo con un otro (Filloux, 2012). Llevada al campo de la formación pre profesional en nuestra investigación, nos interesamos por estudiar la vinculación que el sujeto establece con el proceso formativo general y con las diferentes instancias de evaluación de los aprendizajes. Los cursos del estudio empírico se ubican en los últimos tramos de las carreras de grado y sitúan al estudiante, de diferentes maneras, con la profesión. Estas situaciones requieren la movilización y puesta en práctica de saberes y habilidades construidas a lo largo de la carrera en un proceso que implica, además, representaciones, deseos y expectativas sobre su futuro rol y campo profesional. Esta complejidad nos llevó a considerar, en la instancia inicial de definición del objeto, la necesidad de abordar al sujeto de la formación desde las dimensiones tanto cognitiva (Doyle, 1983) como psíquica-emocional (Bion, 1980).

Desde una perspectiva cognitiva, entendemos que el sujeto construye conocimiento a partir de la interacción con el objeto de saber. Esa interacción habla de una vinculación entre sujeto y objeto que no es lineal ni causal, por el contrario, implica una serie de mediaciones. Algunas de ellas remiten a la actividad mental del sujeto; otras a la mediación producto del pensamiento del profesor. Para caracterizar esta perspectiva, tomamos los aportes de Doyle (1983). Este autor postula que el trabajo académico de los estudiantes se define a partir de tareas académicas que dirigen el pensamiento y la acción, y regulan las estrategias de selección y procesamiento de la información. De acuerdo con este planteo, los alumnos aprenderán aquello que la tarea los Ileve a realizar, es decir, las demandas de aprendizaje que ella implique. La demanda de aprendizaje es definida como un tipo de situación del medio ambiente escolar donde los alumnos deben interpretar ciertos indicadores del aula para resolver las tareas académicas exitosamente. 
De Prácticas y discursos

Universidad Nacional del Nordeste

Centro de Estudios Sociales

Año 7, Número 9, 2018, Marzo

ISSN 2250-6942
El lugar de la Teoría en la construcción del objeto de Investigación.

Decisiones teốricas que involucran un cambio epistemológico

The place of Theory in the construction of the Research object. Theoretical decisions involving an epistemological change

El abordaje del sujeto de la formación implica considerar, también, los aspectos subjetivos. Desde esta dimensión es posible advertir la presencia de deseos, temores, ansiedades y frustraciones, así como también proyecciones e identificaciones con el sujeto formador. El análisis de la vida psíquica de la clase puede complejizarse al considerar la relación emocional que el sujeto establece con el conocimiento a partir de una situación de frustración inicial y su tolerancia o intolerancia a no conocer el objeto en forma completa. Los modos de transitarla permitirán establecer con el objeto de conocimiento diferentes vinculaciones. Al respecto, señala Bion (1980) que conocer supone un vínculo emocional con el objeto de conocimiento. Esta situación se encuentra siempre signada como una experiencia dolorosa debido al esfuerzo de postergación que implica aceptar que el conocimiento verdadero y total del objeto de saber no es posible. Frente a aquella frustración, entonces, puede haber dos vías: tolerarla o evitarla. La primera es la que da lugar a modificaciones que producen el pensamiento y el conocimiento. La segunda, de evitación de la frustración, implica la evasión y la eliminación de elementos que no pueden ser procesados. Esa imposibilidad de tolerar la frustración puede llevar a fantasear que se sabe o rechazar y negar el conocimiento y evitarlo.

El aprender de la experiencia emocional (Bion, 1980) supone lograr una abstracción de esa misma experiencia, una representación de la emoción que implica transformar y elaborar esas emociones en objetos "digeribles" para el psiquismo. En otros términos, aprender de la experiencia emocional requiere poder hacer pensable la emoción.

Estas nociones permitieron definir la siguiente pregunta de investigación: ¿Cómo se caracteriza la relación que el sujeto establece con la situación de formación en general y con la instancia de evaluación en particular a partir de aspectos tales como: las habilidades cognitivas implicadas en el desempeño en la situación, la vinculación emocional establecida, las representaciones sobre la formación, la evaluación y la profesión como práctica futura del estudiante? Se trata de un interrogante que permite pensar al sujeto desde una mirada que incluye tanto lo cognitivo como lo emocional.

\section{CONCLUSIONES}

Este artículo ha descripto y puesto de manifiesto el lugar de la teoria en la construcción y redefinición del objeto de estudio y 
De Prácticas y discursos

Universidad Nacional del Nordeste

Centro de Estudios Sociales

Año 7, Número 9, 2018, Marzo

ISSN 2250-6942

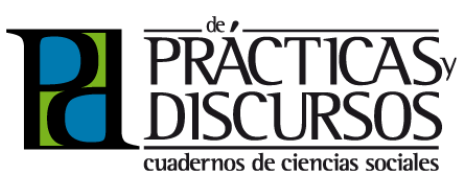

las preguntas de investigación como posibilitadoras de lo que Najmanovich (2008:20) Ilama "hacer aparecer nuevos planos de realidad para explorar y enriquecernos", en el marco del enfoque de la complejidad. Esta reflexión metodológica y epistemológica se realizó desde una investigación doctoral sobre la evaluación de los aprendizajes en instancias de formación pre profesional, sus vinculaciones con la profesión y el proceso formativo del sujeto. Se trata de una reflexión situada, tanto desde lo epistemológico como en lo metodológico, en el enfoque clínico (Souto, 2010b; Mazza, 2015) que asume, como uno de sus rasgos constitutivos, el trabajo sobre la implicación del sujeto investigador (Barbier, 1977; Ardoino, 1997). Ello implica poner en análisis sensaciones, impresiones y pensamientos conscientes e inconscientes propios del investigador, tarea que se realiza siempre con la mediación de otro, en este caso de la directora de la tesis de doctorado. Desde su rol, interpreta aspectos subjetivos e inconscientes que se registran en los diferentes momentos del proceso de investigación y ayuda develarlos y poner en visibilidad el propio posicionamiento del investigador en relación con su objeto de estudio.

La definición inicial del objeto de estudio estuvo dada a partir de la construcción de preguntas que surgieron durante el proceso de análisis y elaboración de conclusiones realizadas en una tesis de Maestría en Didáctica sobre la evaluación de los aprendizajes mediante casos y problemas auténticos, y su vinculación con las modalidades de estudio de los alumnos. Debido a la relevancia que adquirió en ese trabajo la teoría de Evaluación Auténtica (Wiggins, 1990), decidimos analizar sus presupuestos epistemológicos y teóricos. En ese análisis encontramos una orientación hacia un tipo de evaluación sobre la base de tareas complejas referidas tanto a los procesos como a los productos de la evaluación y un modo de pensar al sujeto desde lo cognitivo y racional, y también desde lo actitudinal, dejando ver de esta manera un sujeto que siente junto al pensar. Este análisis favoreció la toma de conciencia sobre la necesidad de adoptar un marco teórico con posicionamiento epistemológico explícito.

La lectura de teorias e investigaciones sobre la temática de estudio, tanto dentro del campo de la didáctica como desde otras disciplinas, permitió ampliar la mirada sobre el objeto y, con ello, orientar la redefinición de las preguntas de investigación. Este camino permitió "habilitar otros interrogantes, gestar otra mirada" (Najmanovich, 2008:20) en un sentido epistemológico y metodológico a la vez. 
De Prácticas y discursos

Universidad Nacional del Nordeste

Centro de Estudios Sociales

Año 7, Número 9, 2018, Marzo

ISSN 2250-6942
El lugar de la Teoría en la construcción del objeto de Investigación.

Decisiones teóricas que involucran un cambio epistemológico

The place of Theory in the construction of the Research Object. Theoretical decisions involving an epistemological change

En el inicio, partimos de preguntas que focalizaban en aspectos técnico-instrumentales de la tarea de evaluación, en su vinculación con la formación para la profesión en la universidad y en una mirada basada en los aspectos cognitivos del proceso de aprendizaje de los estudiantes.

La lectura de otras teorias y diversos trabajos de investigación permitió situarnos en una perspectiva de la formación entendida como trabajo sobre uno mismo (Ferry, 2008). En este recorrido también comprendimos a la formación pre profesional en el entramado de los mundos de la enseñanza, la formación y la profesión (Barbier, 1999; Barbier y Galatanu, 2004), y desde la noción de artificiosidad (Souto, 2010a).

Desde la sociología de las profesionales, entendimos a la profesión como fenómeno sociocultural situado históricamente y sometido a cambios de la vida política, económica, social y cultural (Souto y otros, 2010), y como espacios estructurados de posiciones ocupadas por miembros que pugnan por posiciones de poder (Bourdieu, 1990).

Tomamos aportes de la sociología de la educación (Perrenoud, 2008) y de la didáctica (Camilloni, 2012; Cols, 2009) para comprender la evaluación en un marco social, institucional y profesional docente; hemos situado las lógicas antagónicas que forman parte de las decisiones políticas, éticas y pedagógicas implicadas en la evaluación; señalando, además, su carácter crítico en la universidad como parte del proceso de certificación de competencias para el ejercicio profesional.

Finalmente, consideramos perspectivas psicológicas de corte cognitivo (Doyle, 1983) y psicoanalítico (Bion, 1980) para pensar al sujeto de la formación. Desde esas líneas, partimos del supuesto según el cual la formación pre profesional moviliza tanto aprendizajes técnicos como deseos, temores y ansiedades, expectativas sobre la formación, la evaluación y la profesión.

Luego de haber realizado este recorrido, nuestro interés sobre el objeto de estudio quedó redefinido considerando lo señalado a continuación. Desde una dimensión socioprofesional, nos interesamos por analizar la relación entre la propuesta y dispositivos de formación pre profesional universitaria, las características del campo profesional y los requerimientos de desempeño para el desarrollo de la profesión. La dimensión pedagógica-didáctica nos orienta en la comprensión de los fundamentos de la propuesta y dispositivos de formación y de evaluación de los 
De Prácticas y discursos

Universidad Nacional del Nordeste

Centro de Estudios Sociales

Año 7, Número 9, 2018, Marzo

ISSN 2250-6942

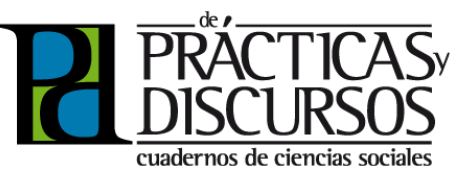

aprendizajes empleados, junto con las concepciones acerca de la práctica y del saber profesional en cada caso. La dimensión instrumental permite analizar los tiempos, espacios, tareas, actividad, tipos de instrumentos de evaluación, demanda cognitiva, recursos de la formación. Finalmente, desde la dimensión del sujeto de la formación, nos acercamos a comprender los procesos cognitivos demandados por las tareas de la formación, los modos de vinculación emocional con el conocimiento y las representaciones de los estudiantes sobre la situación de formación, sobre la evaluación y la práctica profesional futura.

Esta reflexión metodológica y epistemológica ilustró cómo ciertas tradiciones disciplinarias del campo de la didáctica permitieron construir objetos posibles de ser investigados -en tanto formaciones discursivas que posibilitan la constitución de saberes-, operando así la idea según la cual "una vez establecida el área disciplinaria (...) afectará las lecturas posteriores" (Sautu, 2003:25). En el estudio que dio lugar a la tesis de Maestría y el inicio de la investigación doctoral, se trabajó desde una perspectiva centrada en los aspectos técnico-estructurales de la tarea de evaluación y en una concepción del aprendizaje y del sujeto desde sus aspectos racionales y cognitivos, como hemos ya mencionado. No obstante, reconocer la validez de mirar la evaluación desde esas teorías, nos habilitó también a mirarla con nuevos ojos, tomando la metáfora de Najmanovich (2008) para referirse al enfoque de la complejidad. En este marco, consideramos relevante tomar teorías y conceptos provenientes de otros campos disciplinarios como la sociología de las profesiones, la didáctica profesional, el psicoanálisis, entre otros.

Al enfatizar las relaciones entre saber y poder, dando cuenta del primero como una manera histórica de mirar y ordenar el mundo desde una arqueología del saber, Foucault (1970) nos ayuda a pensar cómo ciertas tradiciones de investigación y marcos teóricos constituidos como dominantes y hegemónicos -en este caso dentro de la didáctica- hicieron pensables ciertos objetos y no otros dentro del recorrido por la investigación al que nos hemos referido en este artículo. Tal como señala Foucault (1970), el hecho de que algunas formaciones discursivas puedan tornarse saberes que devengan luego saberes científicos da cuenta de que ciertos objetos de estudio puedan ser pensados como campo de enunciaciones posibles. Es posible pensar este devenir en relación con la adopción de un enfoque de multirreferencialidad 
De Prácticas y discursos

Universidad Nacional del Nordeste

Centro de Estudios Sociales

Año 7, Número 9, 2018, Marzo

ISSN 2250-6942
El lugar de la Teoría en la construcción del objeto de Investigación.

Decisiones teóricas que involucran un cambio epistemológico

The place of Theory in the construction of the Research object. Theoretical decisions involving an epistemological change

teórica (Ardoino, 1993), que habilitó construir y hacer pensable la realidad y los problemas de investigación desde otras enunciaciones posibles.

Tal como hemos señalado, estos cambios teóricos y epistemológicos en el modo de abordar la investigación sobre la evaluación representan una variación respecto de las maneras de mirarla desde investigaciones previas, las que, generalmente, la estudian desde una perspectiva didáctica de base constructivista y cognitivista; perspectiva que enfatiza una mirada del sujeto investigador desde los aspectos técnicos del instrumento y el formato de evaluación, y concibe al sujeto de aprendizaje desde sus aspectos cognitivos y racionales. El tránsito experimentado en el proceso de redefinición del objeto evaluación de los aprendizajes a partir de la búsqueda y empleo de otras teorías, como maneras de mirar el mundo con nuevos ojos (Najmanovich, 2008), permitió advertir diferentes dimensiones sobre ese objeto de estudio y, de esta manera, complejizar la mirada de la evaluación de los aprendizajes y los procesos de formación pre profesional. Esta redefinición posibilitó resituarla en sus aspectos instrumentales -los que no se niegan ni dejan de lado- junto con otros de orden socioprofesional, pedagógicodidáctico y del sujeto de la formación.

Señalamos, finalmente, que concebimos a esta reflexión metodológica sobre el trabajo de investigación como parte del proceso de formación dada en una transición entre los estudios de doctorado y la construcción del oficio de investigador.

\section{REFERENCIAS}

AFPA (1992). Fomation professionnelle. Vocabulaire des formateurs. Paris: Afpa.

AHUMANDA, P. (2005). La evaluación auténtica: un sistema para la obtención de evidencias y vivencias de los aprendizajes. Perspectiva Educacional, Formación de Profesores, Vol. 45, 1124. Valparaiso, Chile.

ANDREOZZI, M. (2006). Encuadres y dispositivos de formación en la práctica profesional: Una aproximación clínica a los procesos de acompañamiento de los estudiantes. Tesis (Maestria en Formados de Formadores). Facultad de Filosofía y Letras, Universidad de Buenos Aires (inédito). 
ARDOINO, J. (1993). L'approche multiréférencielle (pluriel) des situations éducatives et formatives. Pratiques de Formationanalyses, 25-26, Avril.

(1997, 4 DE NOVIEMBRE). La implicación. Conferencia impartida en el Centro de Estudios sobre la Universidad. México: Unam.

ARDOINO, J. Y BERGER, G. (1998). La evaluación como interpretación. México: Universidad Iberoamericana.

BARBIER, J.M. Y GALATANU, 0. (2004). Les savoirs d'action: una mise en mot des compétences? Cap. 2: Savoirs, capacites, compétences, organisation des champs conceptueles (Trad. Sibila Núñez). Paris: L'Harmattan.

BARBIER, J.M. (1999). Prácticas de formación. Evaluación y análisis. Colección Formador de Formadores. Serie Los Documentos. Buenos Aires: Facultad de Filosofía y Letras, UBA-Novedades Educativas.

BARBIER, R. (1977). La recherche-action dans l'institution educative. Paris: Gauthiers Villars.

(2001). Los límites de la competencia. El conocimiento, la educación superior y la sociedad. Barcelona: Gedisa. Bion, W. (1980). Aprendiendo de la experiencia. Buenos Aires: Paidós.

BOURDIEU, P. (1973). Les marché des biens symboliques. Années Sociologiques, 49-126. Francia, Paris.

(1990). Algunas propiedades de los campos. Sociología y cultura. México: Grijalbo.

CAMILLONI, A. Y COLS, E. (2010). La problemática de la investigación didáctica: El caso de una investigación sobre formatos de evaluación de los aprendizajes y sus relaciones con las modalidades de estudio de los alumnos universitarios (pp. 127-143). En Wainerman, C. y Di Virginia, M. (comps.) El quehacer de la investigación en educación. Buenos Aires: Manantial. 
De Prácticas y discursos

Universidad Nacional del Nordeste

Centro de Estudios Sociales

Año 7, Número 9, 2018, Marzo

ISSN 2250-6942
El lugar de la Teoría en la construcción del objeto de Investigación. Decisiones teóricas que involucran un cambio epistemológico

The place of Theory in the construction of the Research object. Theoretical decisions involving an epistemological change

CAMILLONI, A.; COLS, E.; BASABE, L.; AMANTEA, A. Y DI MATTEO, M.F. (2012). Informe final de evaluación proyecto Los formatos de evaluación de los aprendizajes y sus relaciones con las modalidades de estudio de los alumnos universitarios. Programación científica Ubacyt 2008-2010. Argentina: Universidad de Buenos Aires.

COLS, E. (2009). Introducción. La evaluación de los aprendizajes como objeto de estudio y campo de prácticas. Archivos de Ciencias de la Educación, 3(3), 11-14. Argentina, La Plata.

DI MATTEO, M.F. (2013). El uso de casos y problemas auténticos en la evaluación de los aprendizajes en la universidad. Su relación con las disposiciones hacia el aprendizaje y las modalidades de estudio de los alumnos. Tesis (Maestría en Didáctica)-Facultad de Filosofía y Letras, Universidad de Buenos Aires (inédito).

DI MATTEO, M. F. (2014). La formación profesional en la universidad: una propuesta a través de la evaluación mediante casos auténticos. Revista de la Escuela de Ciencias de la Educación, Año10, 9, 271-286. Argentina: Universidad Nacional de Rosario.

DOYLE, W. (1983). Academic work. Review of Educational Research, 53, Summer, 159-199.

FENSTERMACHER, G. (1989). Tres aspectos de la filosofía de la investigación sobre la enseñanza. En Wittrock, M. La investigación de la enseñanza I (pp. 149-177). Barcelona: Paidós.

FERRY, G. (2008). Acerca del concepto de formación. En Ferry, G. Pedagogía de la formación. Serie Los Documentos. Buenos Aires: Facultad de Filosofía y Letras, UBA-Novedades Educativas.

FILLOUX, J.C. (2012). Intersubjetividad y formación. Colección Formador de Formadores. Serie Los Documentos. Buenos Aires: Facultad de Filosofía y Letras, UBA-Novedades Educativas.

FINKELSTEIN, C. (2016). Evaluando las prácticas profesionales durante la formación. InterCambios, 1(3), 33-39.

FLORES, F. (2012, 2 Y 3 DE DICIEMBRE). Dificultades en la 
resolución de consignas de exámenes escritos en estudiantes universitarios. III Jornadas Nacionales y I Jornadas Latinoamericanas de Investigadores en Formación en Educación, Universidad de Buenos Aires. Buenos Aires (paper).

FOLLARI, R. (2010). Curriculum y doble lógica de inserción: lo universitario y las prácticas profesionales. Cadernos de Pesquisa, 140(40), 529-546, maio/ago.

FOUCAULT, M. (1970). La arqueología del saber. Buenos Aires: Siglo Veintiuno.

GÓMEZ CAMPO, V.M. Y TENTI FANFANI, E. (1993). Universidad y profesiones. Buenos Aires: Miño y Dávila.

HIRST, P. (1997). ¿Qué es enseñar? En Peters, R.S. (ed./comp.) Filosofía de la educación. México: Fondo de Cultura Económica. lesalc-Unesco. (2006). Glosario Regional de América Latina. lesalc-Unesco.

KOLB, D.A. (2001). Experiential learning theory bibliography 1971-2001. Boston: Ma McBer and Co.

LIPSMAN, M. (2004). Nuevas propuestas de evaluación de los aprendizajes en la cátedra universitaria. Análisis de experiencias en el ámbito de la Facultad de Farmacia y Bioquímica de la UBA. Revista del Instituto de Investigaciones en Ciencias de la Educación, año XII, 22, 45-54. Argentina, Buenos Aires.

MARTON, F. Y SALÖ, R. (1976). On qualitative differences in learning I. Outcome and process. British Journal of Educational Psychology, 46, 4-11.

MAZZA, D. (2015). El proceso de construcción de sentido en un enfoque clínico en sentido amplio. Revista Educación, Lenguaje y Sociedad. Instituto para el Estudio de la Educación, el Lenguaje y la Sociedad, 11(11). Argentina: Facultad de Ciencias Humanas, Universidad Nacional de La Pampa.

NAJMANOVICH, D. (2008). Mirar con nuevos ojos. Nuevos paradigmas en la ciencia y pensamiento complejo. Buenos Aires: Editorial Biblos. 
De Prácticas y discursos

Universidad Nacional del Nordeste

Centro de Estudios Sociales

Año 7, Número 9, 2018, Marzo

ISSN 2250-6942
El lugar de la Teoría en la construcción del objeto de Investigación. Decisiones teóricas que involucran un cambio epistemológico

The place of Theory in the construction of the Research object. Theoretical decisions involving an epistemological change

PERRENOUD, P. (2008). La evaluación de los alumnos. De la producción de la excelencia a la regulación de los aprendizajes. Buenos Aires: Ediciones Colihue.

SAUTU, R. (2003). Todo es teoría. Objetivos y métodos de investigación. Buenos Aires: Lumiere.

SOUTO, M. (2010A). El carácter de "artificio" del dispositivo pedagógico en la formación para el trabajo. Buenos Aires: OPFyL.

SOUTO, M. (2010B). La investigación clínica en Ciencias de la Educación. Revista del Instituto de Investigaciones en Ciencias de la Educación, 32(XVII), 57-74.

SOUTO, M. Y OTROS (2010). Informe final y resultado de proyecto de investigación La formación para el trabajo en carreras universitarias y el análisis de los dispositivos de formación preprofesional, programación científica Ubacyt 2008-2010.

WIGGINS, G. (1990). The case for authentic assessment. Practical Assessment, Research \&t Evaluation, 2(2). 